\title{
TURBULENCE IN MAIN SEQUENCE STARS
}

\author{
Thomas Gehren \\ Max-Planck-Institut für Astronomie \\ Königstuh1, 6900 Heidelberg, Germany
}

\section{Introduction}

It has for long been realized that the interpretation of stellar spectral lines normally requires broadening velocities well in excess of purely thermal motions. The origin and structure of such velocity fields, which have usually been summarized as turbulence, still appear to be a subject of controversy.

In the sun, according to worrall and wilson (1973), there is no evidence for small scale velocity fields with amplitudes large enough to account for the observed line broadening. Subsequent1y, Wilson and Guidry (1974) tried to explain the center-to-limb variation of the solar $\mathrm{Na} D$ lines by temperature fluctuations associated with a twostream model. More recently, Nelson and Musman (1977) have constructed a model of the solar granulation which predicts granular motions with a scale height appreciably smaller than indicated by the unresolved small scale velocities in the solar photosphere. As Deubner (1976) has shown, further out in the solar chromosphere short period acoustic waves may account for the observed line broadening velocities.

The acoustic flux generated in the convection zone of main sequence stars has been estimated by Hearn (1974) to be insufficient to maintain microturbulence against viscous dissipation, if turbulence is due to progressive sound waves. His suggestion that the broadening of spectral lines results from convective overshoot into the photosphere has been investigated in detail by Nordlund (1978), who confirms that even a laminar velocity field describing the up- and downward motions of penetrating convective eddies can reproduce 1 ine strengths as well as their center-to-limb variation in the solar photosphere. The amplitude of such a velocity field is chosen to have a maximum on granular scales, and Nissen and Gustafsson (1978) suggest that parametrization of small scale velocity fields derived from analysis of horizontally homogeneous model atmospheres probably leads to an overestimate of the true microturbulence. 


\section{Micro- and Macroturbulence}

The two-component model atmosphere approach has not yet been systematically applied to the interpretation of spectral lines in main sequence stars. Except for the Sun, our knowledge of stellar velocity fields is restricted by the assumption of plane-parallel horizontally homogeneous atmospheres in which the concept of random motions on two different scales (micro- and macroturbulence) is used.

Small scale motions are usually described by a gaussian velocity distribution, where the microturbulence parameter $\xi$ represents the most probable amplitude. Some authors have tried to derive its dependence on continuum optical depth, $\xi(\tau)$, from the application of the Goldberg method. For procyon (F5IV-V) Sikorski (1976) obtained a microturbulence parameter smoothly increasing from $2.5 \mathrm{~km} / \mathrm{s}$ at $\tau=0.3$ to $3.5 \mathrm{~km} / \mathrm{s}$ at $\tau=0.001$. A similar increase with height, derived for Arcturus, is in accordance with the work of Mäckle et al. (1975). Contradicting these results, Stenholm (1977) has determined $\xi(\tau)$ for the sun and the subgiants $\eta$ Cep (KOIV) and $y$ Cep (KIIV) using Goldberg's method. His solar microturbulence shows a distinct decrease from $1.5 \mathrm{~km} / \mathrm{s}$ at $\tau=0.3$ to $0.5 \mathrm{~km} / \mathrm{s}$ at $\tau=0.001$, and a similar behaviour of $\xi(\tau)$ is found for $\eta$ and $\gamma$ Cep.

It has been emphasized by Holweger et al. (1978) that the horizontally homogeneous approach requires the existence of an anisotropic small scale velocity field in the sun, since the limb strengthening of photospheric lines cannot be explained entirely by a depth-dependent isotropic parameter. Moreover, a solar microturbulence, increasing with height in the fashion derived for Procyon, is incompatible with the observed width of faint lines originating in the upper photosphere at $\tau<0.01$. These lines require very small velocity amplitudes, probably below $0.5 \mathrm{~km} / \mathrm{s}$ (cf. Canfield and Beckers, 1976, for a review on unresolved solar motions). Thus, the most plausible depth variation seems to be represented by a smooth decrease of photospheric small scale velocities with height, followed by an increase in chromospheric layers.

Large scale motions (macroturbulence) in the atmosphere of Procyon have been estimated by Evans et al. (1975). From a line shape analysis they rule out microturbulence velocities larger than 1 to $2 \mathrm{~km} / \mathrm{s}$, the dominant line broadening process being large scale motions with most probable velocities of about $2 \mathrm{~km} / \mathrm{s}$. This agrees roughly with the ratio of micro/macro amplitudes in the sun derived by Holweger et al. (1978) as well as with that in giants (Reimers, 1976). 
III. Microturbulence and Basic Stellar Parameters

Further information on small scale velocity fields in stars along the main sequence is constrained by the assumption of an isotropic gaussian velocity distribution with a depth-independent microturbulence parameter $\xi$. The determination of the microturbulence parameter has been carried out according to one of the following methods:

(a) Narrow band photometry, calibrated with synthetic spectra from model atmospheres (Gustafsson et al., 1974, Nissen and Gustafsson, 1978)

(b) Curve-of-growth analyses of different degrees of sophistication as compiled by Glebocki (1973) and Morel et al. (1976)

(c) Fourier transform analysis of line profiles (Gray, 1973)

Since microturbulence values taken from the catalogues of Glebocki and Morel et al. are often contradictory, the principal sources of systematic errors must be elucidated.

Frequently the microturbulence is only a by-product of abundance analyses, and some authors do not seem to have recognized that their element abundances are severely degraded by an improper determination of the turbulence parameter. It should be emphasized that the onelayer curve-of-growth analysis is particularly unsuited to derive small scale velocities, because the choice of the "best fit" theoretical curve is often biased by the large scatter in weak line strengths. Curve-of-growth results may additionally be affected by unrecognized blends and uncertainties in the determination of the continuum level.

Besides these general statements some sources of systematic errors deserve a more detailed investigation (cf. Gray and Evans, 1973):

(1) Effective temperature and gravity of model atmospheres used to compute saturation parameters or synthetic spectra are usually determined from observed colours by calibration relations. This procedure often involves the sun as a reference point, although the solar colours are a subject of controversy. Frequently the result may have been a significant underestimate of the temperatures of solar-type stars.

(2) The temperature distribution predicted by theoretical lineblanketed model atmospheres of solar-type stars displays strong backwarming effects which cannot be reconciled with observations of the solar continuum (Gehren, 1979, see also Fig. 2 of Gustafsson and Bell, 1979). The model temperature gradients in the region of line formation are too steep and, when applied to the interpretation of observed line strengths, may simulate a low microturbulence.

(3) Many abundance analyses have been based on absolute oscillator strengths. Because of systematic errors in these measurements prior to 
1970, most of the related microturbulence data are unreliable (Reimers, 1976). A rediscussion by Andersen (1973) revealed a considerable change of the earlier microturbulence determinations.

(4) The problem of absolute f-values may be overcome by analyses using solar f-values. However, such a procedure requires the knowledge of solar equivalent widths as well as the solar microturbulence parameter (the average small scale velocity amplitude in the solar photosphere). Differential curves-of-growth for long were based on the Utrecht atlas which, as shown by analysis based on modern photoelectric measurements (Gehren, 1978), may lead to a larger scatter of line strengths around the curve-of-growth and to microturbulence values systematically low. Furthermore, broadening velocities obtained for stars depend critically on the solar value. Some stellar data (e.g. Gustafsson et al., 1974) refer to $\xi_{\odot}=0.5 \mathrm{~km} / \mathrm{s}$, as derived by Foy (1972) from a disc center solar curve-of-growth. Recent determinations group around $0.9 \mathrm{~km} / \mathrm{s}$ for the disc center and $1.3 \mathrm{~km} / \mathrm{s}$ for the integrated disc (Blackwell et al., 1976, Holweger et al., 1978).

(5) Magnetic intensification and hyperfine structure may play a role in special classes of stars or lines, respectively.

(6) Departures from LTE have been shown to be of minor importance as far as line strengths are considered (Holweger, 1973). However, they may affect Fourier transform analysis of line profiles. Smith and Gray (1976) suggest to avoid these difficulties by analyzing lines of different strength. This approach has been challenged by Durrant (1979) who finds that the microturbulence derived from the Fourier transform analysis strongly depends on line strength.

(7) A final source of uncertainty is due to the damping constants (Blackwell et al., 1976). Since the contributions of microturbulence and damping can be separated in a line strength vs. abundance diagram (cf. Garz et al., 1969, Fig. 1), however, correction factors for the simple hydrogenic treatment of van der wals interaction may be found.

From this discussion it is evident that, in order to study microturbulence, we have to restrict ourselves to results presumably unaffected by systematic errors. The data compiled by Glebocki (1973) and Morel et al. (1976) have been disregarded on this account. The microturbulence values displayed in Figs. 1 and 2 are from the following sources: narrow band photometry of Nissen and Gustafsson $(1978,0)$, curve-of-growth analyses of Chaffee (1970, corrected by Andersen, $1973,+)$ and Clegg $(1977, \times)$, model atmosphere analyses of Gehren (1977, $\Delta, 1978,1979, *)$, and Fourier transform analysis (Gray, 1973, 口). Small symbols denote stars for which contradictory results have been found 

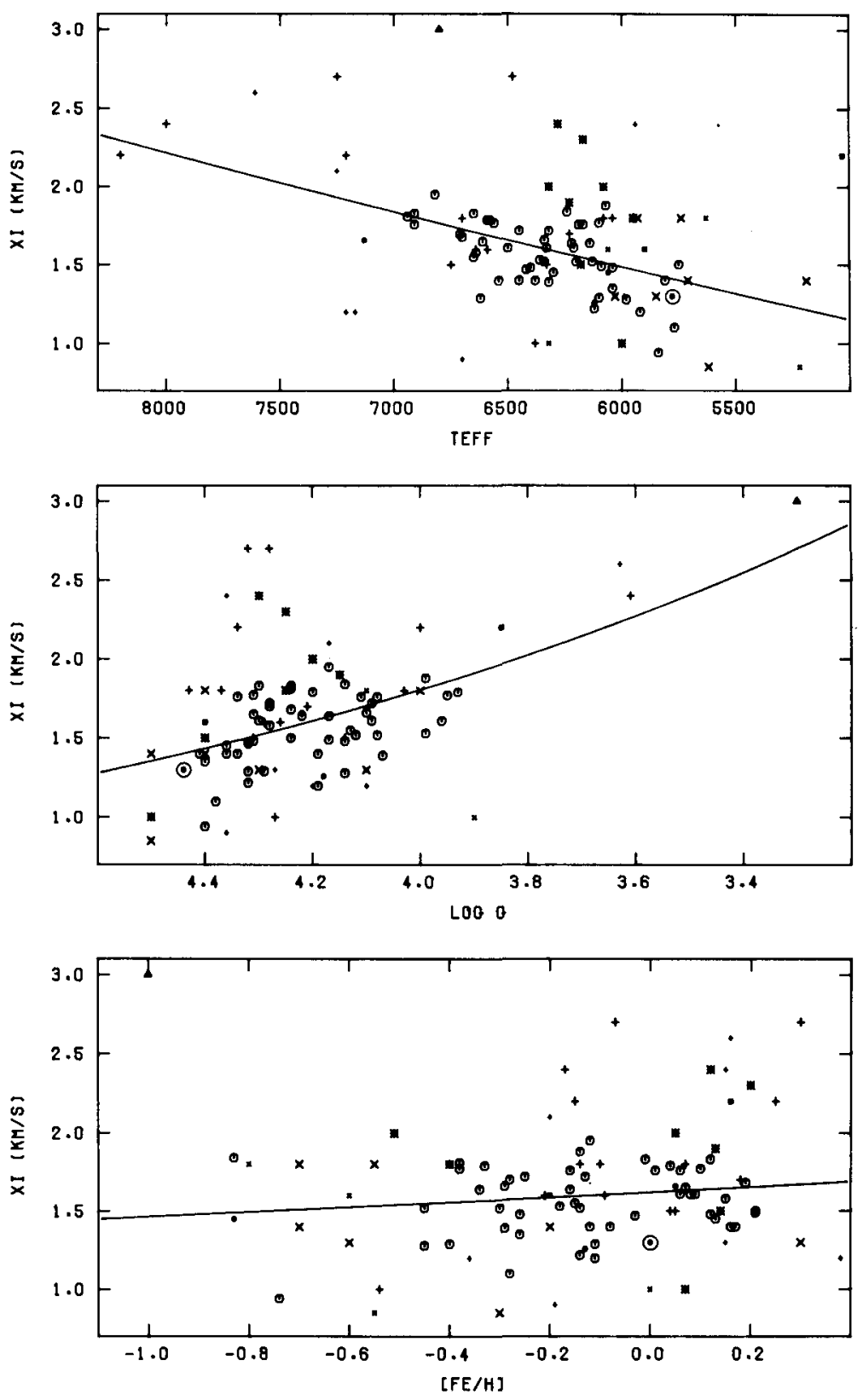

Figure 1: Microturbulence velocities as a function of the basic stellar parameters, temperature, gravity, and metal abundance. Solid curves represent least square power laws. See text for an explanation of the symbols 
among the sources just mentioned and the compilations of Glebocki and Morel et al. The corresponding microturbulence values have been given lower weight.

In Fig. 1 is shown the degree of correlation between microturbulence and the three basic stellar parameters, $\mathrm{T}_{\text {eff }}$, log $\mathrm{g}$, and $[\mathrm{Fe} / \mathrm{H}]$, where $[\mathrm{Fe} / \mathrm{H}]$ is the logarithmic metal abundance relative to the Sun and $(\mathrm{Fe} / \mathrm{H})=\exp _{10}[\mathrm{Fe} / \mathrm{H}]$. Since for some of the stars either gravity or metal abundance is not given by the authors, these data have been estimated from calibration relations of $\delta c_{1}$ and $\delta m_{1}$ (cf. Nissen and Gustafsson, 1978). Least square solutions assuming a power law dependence yield

$$
\begin{aligned}
& \xi=8.610^{-6} \mathrm{~T}_{\text {eff }}^{1.386} \pm 0.352 \mathrm{~km} / \mathrm{s}, \\
& \xi=18 \cdot \mathrm{g}^{-0.249 \pm 0.345 \mathrm{~km} / \mathrm{s}, \text { and }} \\
& \xi=1.6(\mathrm{Fe} / \mathrm{H})^{0.044 \pm 0.395 \mathrm{~km} / \mathrm{s},}
\end{aligned}
$$

while the multivariate solution

$$
\xi=2.510^{-3} \mathrm{~T}_{\text {eff }}^{0.945} \mathrm{~g}^{-0.175}(\mathrm{Fe} / \mathrm{H})^{0.023} \pm 0.344 \mathrm{~km} / \mathrm{s}
$$

is only a marginal improvement.

The considerable scatter displayed in Fig.1 is due to a few stars with comparatively high microturbulence, as $\beta$ Vir, $9 \mathrm{Com}, 37 \mathrm{UMa}$ and $\mu$ Her A. Since for $\beta$ Vir and 9 Com the error in the determination of $\xi$ is probably within $\pm 0.3 \mathrm{~km} / \mathrm{s}$ (Gehren, 1978, 1979), it is felt that there are significant departures. from the mean relation that deserve special explanation. An independent determination with narrow band photometry would be also desirable in those cases. Excessively high velocities for A-type stars as noted by Baschek and Reimers (1969) and Smith (1971) are probably the result of using inaccurate absolute fvalues (cf. Andersen, 1973). The same holds for the Hyades dwarfs of Chaffee et al. (1971). The Hyades stars VB 14 and VB 47 included here do not show a high microturbulence.

Whereas least square solutions confined to the more homogeneous data of Nissen and Gustafsson (1978) yield a considerable reduction of the scatter, the functional dependence found above is not changed. Thus the least square solutions are not excessively weighted by the few stars with high temperature or low gravity. From Fig. 1 it is seen that the microturbulence velocity is not an independent stellar parameter, in agreement with the results of Nissen and Gustafsson. 


\section{Discussion}

The overall variation of the microturbulence parameter with $T_{\text {eff }}$ and $\log \mathrm{g}$ is remarkably similar to the one found by Reimers (1973) for giants and supergiants covering a considerably larger interval in gravity. Moreover, it closely follows the temperature and gravity dependence of the wilson-Bappu effect, which yields for the Ca II $K$ emission widths $W_{O} \sim \mathrm{T}_{\text {eff }}^{1.25} \mathrm{~g}^{-0.22}$. This seemingly uniform behaviour of stellar velocity fields, extending over a large part of the Hertzsprung-Russell diagram, led Böhm-Vitense (1975) and Reimers (1976) to suggest that microturbulence is correlated with the velocities in the upper layers of stellar convection zones. Even within the restricted range in temperature and gravity considered in Fig.1, a correlation between microturbulence and convective velocities does indeed exist. This is shown in Fig. 2 where we have drawn microturbulence velocities against maximum convective velocities computed by de Loore (1970), since photospheric velocities due to penetrative convection are not yet available for a grid of temperatures and gravities. Although the maximum convective velocities depend critically on the mixing-length parameter, their variation with $\mathrm{T}_{\text {eff }}$ and log $\mathrm{g}$ appears to be reasonably well defined. The linear least squares solution shown in Fig. 2 is

$$
\xi=0.31 v_{\max }+0.79 \pm 0.328 \mathrm{~km} / \mathrm{s}
$$

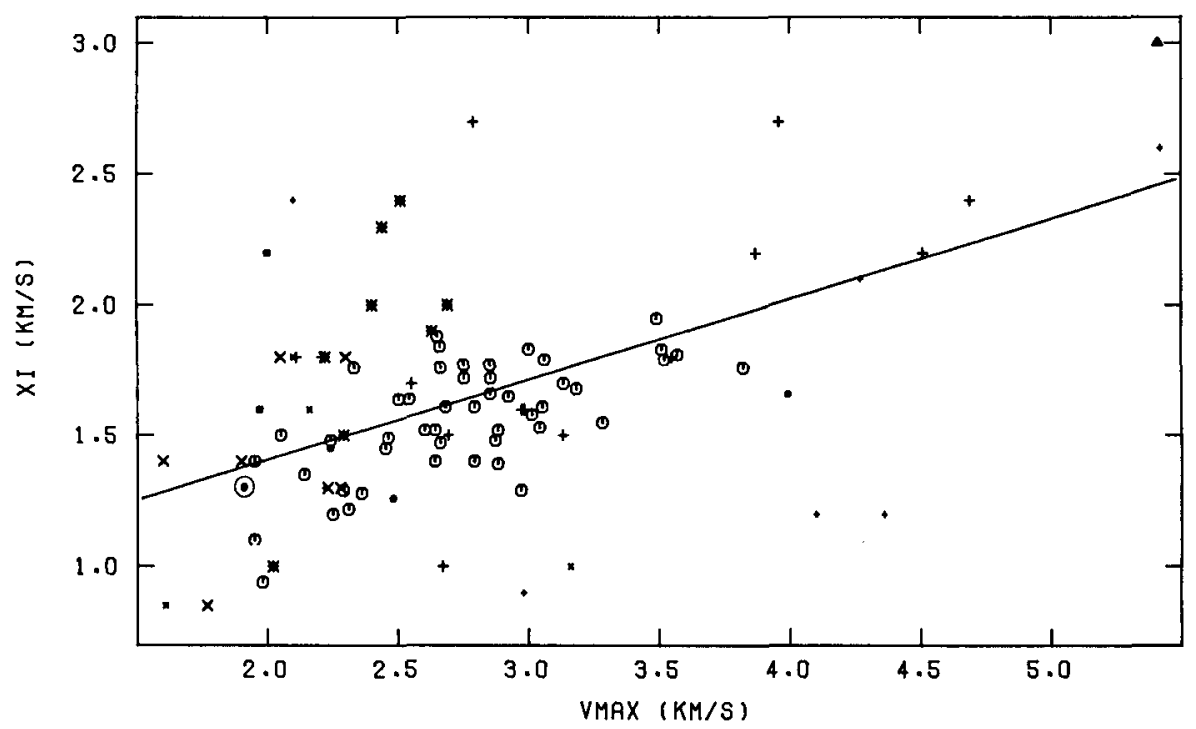

Figure 2: Microturbulence vs. maximum convective velocities, computed by de Loore (1970). The solid line denotes the linear least squares solution 
Since photospheric velocity amplitudes due to convective overshoot may be expected to vary smoothly with maximum convective velocities, it is perhaps not surprising that this solution corresponds to

$$
\xi \simeq v_{\text {conv }}(\tau=1)
$$

when $v_{\text {conv }}(\tau=1)$ and $v_{\max }$ are taken from Nordlund's two-component models (Nordlund, 1976, Nissen and Gustafsson, 1978). Photospheric velocities of this amplitude may be also derived from mixing-length theory if allowance is made for a non-local computation of velocities (Parsons, 1969, Nordlund, 1974).

Even though the convective origin of the observed broadening velocities seems to be sufficiently well established, the nature of the transport mechanism cannot yet be ascertained. Regular streaming patterns on granular scales may resemble microturbulence as shown by Nordlund (1978). His results are most convincing because they explain the total velocity amplitudes (micro- and macroturbulence) as well as the solar center-to-limb broadening of photospheric lines. There still remains the possibility that a fraction of the convective energy is redistributed to motions on smaller scales $(<100 \mathrm{~km})$, which are unresolvable even in the sun and would look more like the classical microturbulence. Progressive sound waves generated in the convection zone may be another transport mechanism (Edmunds, 1978). However, the corresponding wave velocity amplitudes appear to be too small in the photosphere of a main sequence star. Short period acoustic waves propagating on top of stellar atmospheres are more likely to account for chromospheric motions (Oster and Ulmschneider, 1973, Deubner, 1976).

The similar dependence upon temperature and gravity of both regular convective motions and progressive sound waves provides a simple explanation for the correlation between small scale velocities observed in stellar photospheres and chromospheric Ca II K emission widths.

\section{Concluding Remarks}

In order to discriminate between different line broadening velocity fields, future work will have to concentrate on the sun, where the obtainable spatial and temporal resolution is orders of magnitude higher than for any other star.

A crucial test of the relation outlined here between convective and line broadening velocities máy be supplied by a reexamination of the microturbulence in A-type stars. If convection in main sequence stars fades out beyond effective temperatures of $8500 \mathrm{~K}$, the microturbulence velocities should also diminish. 
The cool branch of the main sequence deserves further attention. No reliable microturbulence values are available for stars later than spectral type G5V.

The microturbulence parameter as a measure of random motions on small scales thus will probably remain one of the main goals of spectrum analysis for years to come.

\section{References}

Andersen, P.H. 1973, Publ.Astron.Soc.Pacific 85, 666

Baschek, B., Reimers, D. 1969, Astron.Astrophys. 2,240

Blackwell, D.E., Ibbetson, P.A., Petford, A.D., Willis, R.B. 1976, Monthly Not.Roy.Astron.Soc. 177,227

Böhm-Vitense, E. 1975, in "Problems in Stellar Atmospheres and Envelopes", eds. B. Baschek, W.H. Kegel, G. Traving, Heidelberg, p. 21

Canfield, R.C., Beckers, J.M. 1976, in "Physique des Movements dans les Atmosphères Stellaires", eds. R. Cayrel, M. Steinberg, Paris, p. 291

Chaffee, F.H. 1970, Astron.Astrophys. 4,291

Chaffee, F.H., Carbon, D.F., Strom, S.E. 1971, Astrophys.J. 166,593

Clegg, R.E.S. 1977, Monthly Not.Roy.Astron.SoC. 181,1

Deubner, F.-L. 1976, Astron. Astrophys. 51,189

Durrant, C.J. 1979, Astron.Astrophys. 76,208

Edmunds, M.G. 1978, Astron.Astrophys. 64,103

Evans, J.C., Ramsey, L.W., Testerman, L.1975, Astron.Astrophys. 42,237 Foy, R. 1972, Astron. Astrophys. 18,26

Garz, T., Holweger, H., Kock, M., Richter, J. 1969, Astron.Astrophys. $\underline{2}, 446$

Gehren, T. 1977, Astron. Astrophys. 59, 303

Gehren, T. 1978, Astron.Astrophys. 65,427

Gehren, T. 1979, Astron. Astrophys. $\underline{75}, 73$

Glebocki, R. 1973, Acta Astronomica 23,135

Gray, D.F. 1973, Astrophys.J. 184,461

Gray, D.F., Evans, J.C. 1973, Journ.Roy.Astron.Soc.Canada 67,241

Gustafsson, B., Kjæergaard, P., Andersen, S. 1974, Astron.Astrophys. 34, 99

Gustafsson, B., Bell, R.A. 1979, Astron.Astrophys. 74, 313

Hearn, A.G. 1974, Astron.Astrophys. 31,415

Holweger, H. 1973, Solar Physics 30,35

Holweger, H., Gehlsen, M., Ruland, F. 1978, Astron.Astrophys. 70,537

de Loore, C. 1970, Astrophys.Space Science $\underline{6}, 60$

Mäckle, R., Holweger, H., Griffin, R., Griffin, R. 1975, Astron. Astrophys. 38,239

Morel, M., Bentolila, C., Cayrel, G., Hauck, B. 1976, in "Abundance Effects in Classification", eds. B. Hauck, P.C. Keenan, Dordrecht, p. 223

Nelson, G.D., Musman, S. 1977, Astrophys.J. 214,912

Nissen, P.E., Gustafsson, B. 1978, in "Astronomical Papers Dedicated to Bengt Strömgren", eds. A. Reiz, T. Andersen, Copenhagen, p.43

Nordlund, \&. 1974, Astron. Astrophys. 32,407

Nordlund, $\AA$. 1976, Astron. Astrophys. 50,23

Nordlund, $\AA$. 1978, in "Astronomical papers Dedicated to Bengt Strömgren", loc.cit.p. 95

Oster, L., Ulmschneider, P. 1973, Astron.Astrophys. 29,1

Parsons, S.B. 1969, Astrophys.J.Suppl. 18,127

Reimers, D. 1973, Astron.Astrophys. 24, 79

Reimers, D. 1976, in "Physique des Movements dans les Atmosphères stellaires", loc.cit.p. 421 
Sikorski, J. 1976, Acta Astronomica 26,1

Smith, M.A. 1971, Astron.Astrophys. 11,325

Smith, M.A., Gray, D.F. 1976, Publ. Astron.Soc.Pacific 88, 809

Stenholm, L.G. 1977, Astron.Astrophys. 61,155

Wilson, A.M., Guidry, F.J. 1974, Monthly Not.Roy.Astron.Soc. 166, 219

Worrall, G., Wilson, A.M. 1973, in "Vistas in Astronomy", ed. A. Beer, Vol. 15, p. 39 